Acta Zoológica Mexicana (n.s.) 23(2): 163-181 (2007)

\title{
USO Y SELECCIÓN DE EMBALSES POR EL PATO MEXICANO ( ANAS DIAZI) EN LA REGIÓN DEL LLANO, AGUASCALIENTES-JALISCO, MÉXICO
}

\author{
Salvador Martín Medina Torres, Marcelo Márquez Olivas y \\ Edmundo García Moya \\ Posgrado en Botánica, Campus Montecillo, Colegio de Postgraduados. \\ Carretera México-Texcoco, Km 36.5. C.P. 56230. Montecillo Estado de México. MÉXICO \\ E-mail: smedinat@colpos.mx.molivas@colpos.mx y edmundo@colpos.mx
}

\begin{abstract}
RESUMEN
Se registró, entre septiembre de 1994 y marzo de 1995, el uso de embalses por el pato mexicano (Anas diazi Ridway, 1886) en la región de El Llano, entre Aguascalientes y Jalisco en México. El objetivo de este estudio fue determinar cuáles embalses son seleccionados o evitados por esta especie, con base en un análisis de uso-disponibilidad de cuerpos de agua. Los resultados sugieren un uso superior al esperado de los bordos, los cuales se utilizan para almacenar agua que se extrae por bombeo para irrigación, durante el otoño y parte del invierno. Este tipo de almacenamiento de agua puede ser importante para la continuidad del ciclo vital del único anátido residente del Altiplano mexicano. La evaluación e identificación de los cuerpos de agua que son utilizados por encima de su disponibilidad, permitiría proponer estrategias para la conservación y aprovechamiento sostenible de esta especie.

Palabras Clave: Pato mexicano, Anas diazi, uso de hábitat, Aguascalientes- Jalisco, México.
\end{abstract}

\begin{abstract}
The use of dams by the Mexican duck (Anas diazi Ridway, 1886) was documented between September 1994 and March 1995, in the region of El Llano, located in Mexico between Aguascalientes and Jalisco. The objective of this study was to determine which type of dam is more frequently used by this species, based on a use-availability water analyses. The results point out a superior use than expected of the reservoirs used as water storage which are pumped for irrigation, throughout fall and part of winter. These dams can be vital for the breeding of ducklings in Mexico. The identification and evaluation of the dams which are more frequently used by the Mexican duck will allow developing strategies for their conservation and sustainable use.

Key words: Mexican duck, Anas diazi, habitat use, Aguascalientes-Jalisco, Mexico.
\end{abstract}

\section{INTRODUCCIÓN}

Anas diazi se conoce en México como pato mexicano o pato triguero y es probablemente una raza del pato de collar (Anas platyrhynchos Ridgway), el más 
común de todos los patos del Hemisferio Norte y ancestro de muchos patos domésticos (Peterson \& Chalif 1989, Kaufman 2005), aunque Aldrich \& Baer (1970) y McCraken et al. (2001) la proponen como una especie diferente. Dicho pato se considera como endémico de México y está en el listado de especies o subespecies amenazadas por la Norma Oficial Mexicana NOM-059-ECOL-2001 (DOF 2002). A este anátido no se le considera una especie migratoria, porque la mayor parte de su población es residente del Altiplano y del centro de México, pero durante el año, puede cambiar de un lugar a otro en busca de embalses para cubrir sus requerimientos de alimentación, reproducción, y refugio. Estos desplazamientos, locales o regionales, son temporales de acuerdo a la disponibilidad de agua y alimento (Williams 1980, Cisneros 1985), o necesidad de otros recursos como una buena cobertura herbácea para anidar (Mellink 1994).

El pato mexicano se distribuye a lo largo de ríos y lagos del Altiplano Central Mexicano y llega hasta Chihuahua, sur de Arizona, Nuevo Mexico y Texas, por la parte oriental de la Sierra Madre Occidental y la cuenca del Río Bravo (Ohlendorf \& Patton 1971, Tomlinson et al. 1973, Palmer 1976, Hubbard 1977, Williams 1980, Cisneros 1985), pero también se le puede encontrar en Nayarit, Jalisco, Michoacán, Estado de México, Distrito Federal, Puebla y Tlaxcala (SEDUE 1989) y posiblemente en otros estados del sur como Oaxaca y Veracruz.

Bellrose (1976) refiere que, en el norte de México y sur de los Estados Unidos, el pato mexicano se aparea durante la primavera y que al inicio del verano, y en ocasiones desde finales de abril y mediados de junio, anida en las orillas de las lagunas y áreas pantanosas, oculto bajo bejucos, juncos y otras malezas (Howell \& Webb 1995). A finales del verano, cuando las crías adquieren el plumaje de los adultos, comienzan a formarse las grandes parvadas de estos anátidos que suelen verse durante el invierno (Bellrose 1976), y, junto con otras aves acuáticas (patos y gansos principalmente), son objeto de aprovechamiento cinegético. Bellrose (1976) cita que durante el invierno el pato mexicano se desplaza desde sus áreas de reproducción, en el norte, hasta 1,000 km hacia el sur. Se estima que alrededor de 500,000 patos y gansos llegan al centro de México cada año durante el invierno; sin embargo, la cantidad es muy variable y poco se conoce sobre sus poblaciones (Perez-Arteaga et al. 2002). La desecación de los humedales para el desarrollo agrícola altera el comportamiento de esas aves y afecta de manera severa a las especies residentes (Bellrose 1976, INE 1994, Leopold 1990, Perez-Arteaga \& Gaston 2004). Arriaga-Cabrera et al. (2000) citan que los humedales de México se están deteriorando de manera constante, ya que más del $95 \%$ son afectados por la erosión y el $80 \%$ por la reducción en las especies de plantas y animales que los integran.

En la región de El Llano, entre Aguascalientes y Jalisco, se inició a principios de 1980 un programa gubernamental de fomento a la agricultura mediante la perforación de pozos y la construcción de un gran número de embalses que en la actualidad 
constituyen la base agrícola del estado (Medina 1996). La infraestructura de irrigación creada para la agricultura modificó el paisaje de la región, característico del Desierto Chihuahuense (Grenot 1983), pero generó hábitats apropiados para el pato mexicano (Williams 1980). Estos embalses podrían ser, por lo tanto, un factor importante para la reproducción y el mantenimiento de sus poblaciones (Leopold 1990, Perez-Arteaga et al. 2002, Riojas-López \& Mellink 2005); sin embargo, dado que varían en cuanto a su tamaño y cantidad, es conveniente analizar cuales son utilizados por el pato mexicano, para inferir sí su uso corresponde o no a su disponibilidad.

La necesidad de determinar la selección o evasión de un hábitat, con relación a su disponibilidad, ha sido reconocida como un primer paso para comprender y explicar mejor las interacciones ecológicas de los organismos con su medio (Neu et al. 1974), pero también podría ser de gran importancia para llevar al cabo acciones orientadas hacia la protección de especies amenazadas y en el manejo de poblaciones silvestres sujetas a explotación constante (Manly et al. 1993). En el caso del pato mexicano, este tipo de análisis constituye un requisito previo para su conservación y manejo exitosos. Peek (1986) cita que el uso del hábitat es sólo su ocupación, pero no considera su posible selección. Por el contrario, Manly et al. (1993) refieren que la selección es el proceso en el cual los animales eligen un recurso en relación con su disponibilidad. Con frecuencia se confunde el término "selección" con "preferencia". En este caso, Manly et al. (1993) consideran que la "preferencia" constituye la verosimilitud con la cual un recurso es seleccionado sí éste permanece disponible de manera equitativa junto con otros. La disponibilidad de dicho recurso, se refiere a la cantidad de éste accesible al mismo individuo o población en un período de tiempo dado. Su abundancia es la cantidad total del mismo presente en el ambiente.

Entre septiembre de 1994 y marzo de 1995, y con el propósito de determinar sí algún tipo de embalse es seleccionado por el pato mexicano, se realizó el presente estudio para analizar el uso de esos cuerpos de agua por dicha especie en la región de El Llano, entre Aguascalientes y Jalisco en México. Con los resultados se busca recomendar acciones de manejo y protección del pato mexicano, para garantizar su sostenibilidad cinegética en el futuro, y promover el uso alternativo del agua en la región.

\section{MATERIALES Y MÉTODOS}

\section{Área de estudio}

La región de El Llano se localiza al sudeste de la Ciudad de Aguascalientes, entre las coordenadas métricas 792249 zona 13 y 197969 zona 14 Oeste, y 2401296 y 2446481 zona 13 Norte. Tiene una superficie de 78,076.19 ha, de las cuales $67.82 \%$ corresponden al estado de Aguascalientes y $32.18 \%$ al de Jalisco (Fig. 1). 
Medina-Torres et al.: Pato mexicano en el Llano

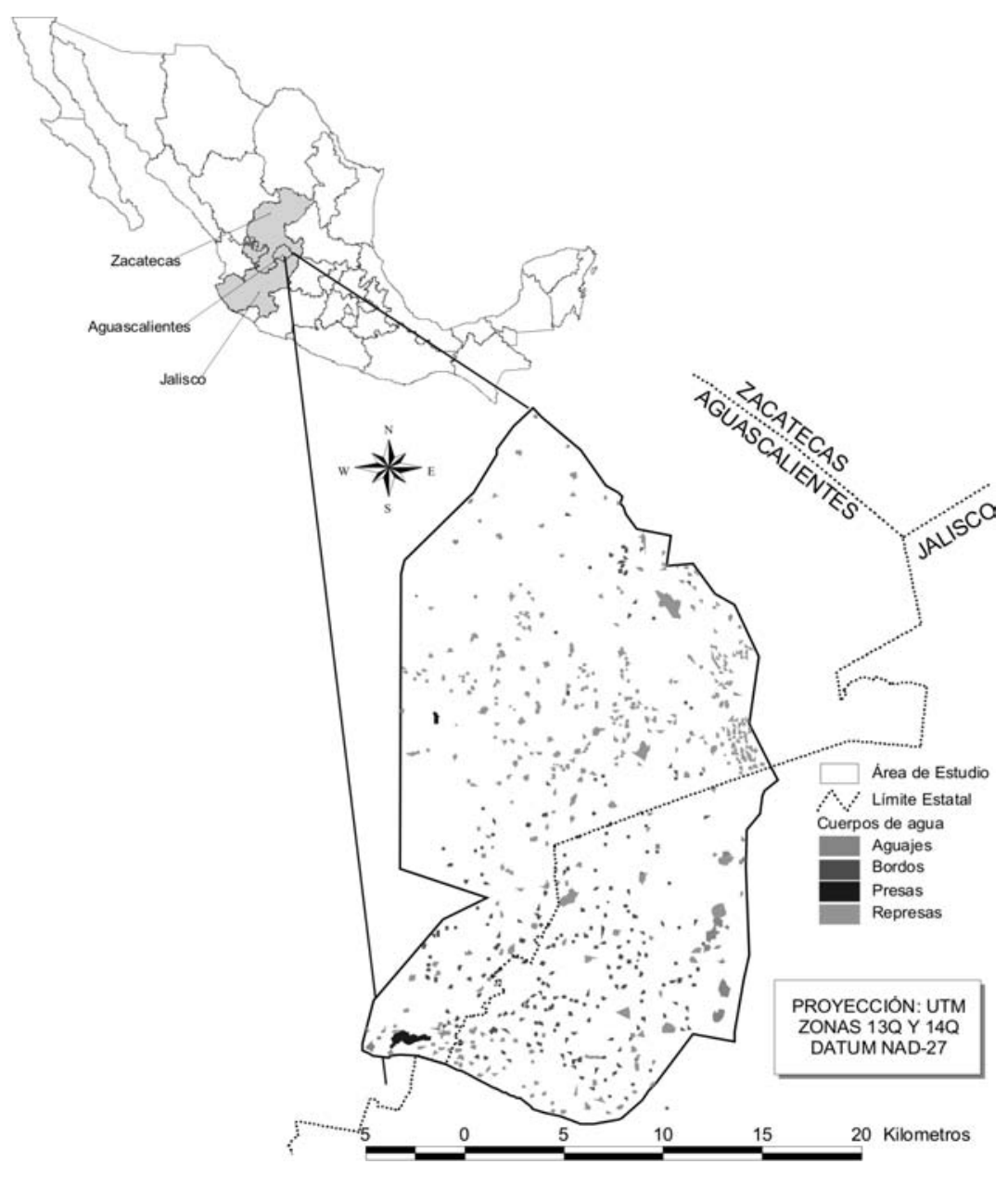

Figura 1

Ubicación del área de estudio y distribución de los cuerpos de agua.

La fisiografía de El Llano es una planicie que se extiende del sudeste de Aguascalientes hacia el noreste de Jalisco, en un gradiente altitudinal que varía de 2,000 a 2,050 m y forma parte de la Provincia de la Mesa Central y de la Sub-provincia de los Llanos de Ojuelos (INEGI 1993). Esta área tiene llanuras extensas de piso consolidado que forman una cuenca de origen volcánico rellena por intercalaciones de material clástico continental de tipo fluvial como conglomerado, arcilla y arena. La vegetación nativa de El Llano ha sido desplazada por el sobre-pastoreo y la agricultura, pero aun persisten algunos remanentes de vegetación original, típica del Desierto Chihuahuense, como los agaves (Agave spp.), las nopaleras (Opuntia spp.), los mesquites (Prosopis spp.), los huizaches (Acacia pp.), y pastizal. El clima en El Llano 
es semiseco-fresco con una temperatura media anual de $18^{\circ} \mathrm{C}$ y precipitación promedio de $500 \mathrm{~mm}$. Los suelos, en su mayor parte, son someros y con un bajo contenido de materia orgánica. Los tipos que predominan en la zona son Planosol eutrico, Xerosol háplico, Planosol mólico, Xerosol lúvico, Feozem háplico y Fluvisol eutrico (INEGI 1981). El uso de la tierra es agrícola (temporal y riego) con algunas áreas de agostaderos.

\section{Cuerpos de agua}

El área a muestrear se determinó mediante el uso de mapas topográficos (escala 1:50,000) y visitas de campo. Con un Sistema de Ubicación Global (GPS, por sus siglas en inglés), se registraron las coordenadas de los embalses para su posterior localización en un mapa topográfico. Con base en la cantidad, distribución (concentración o dispersión) y ubicación

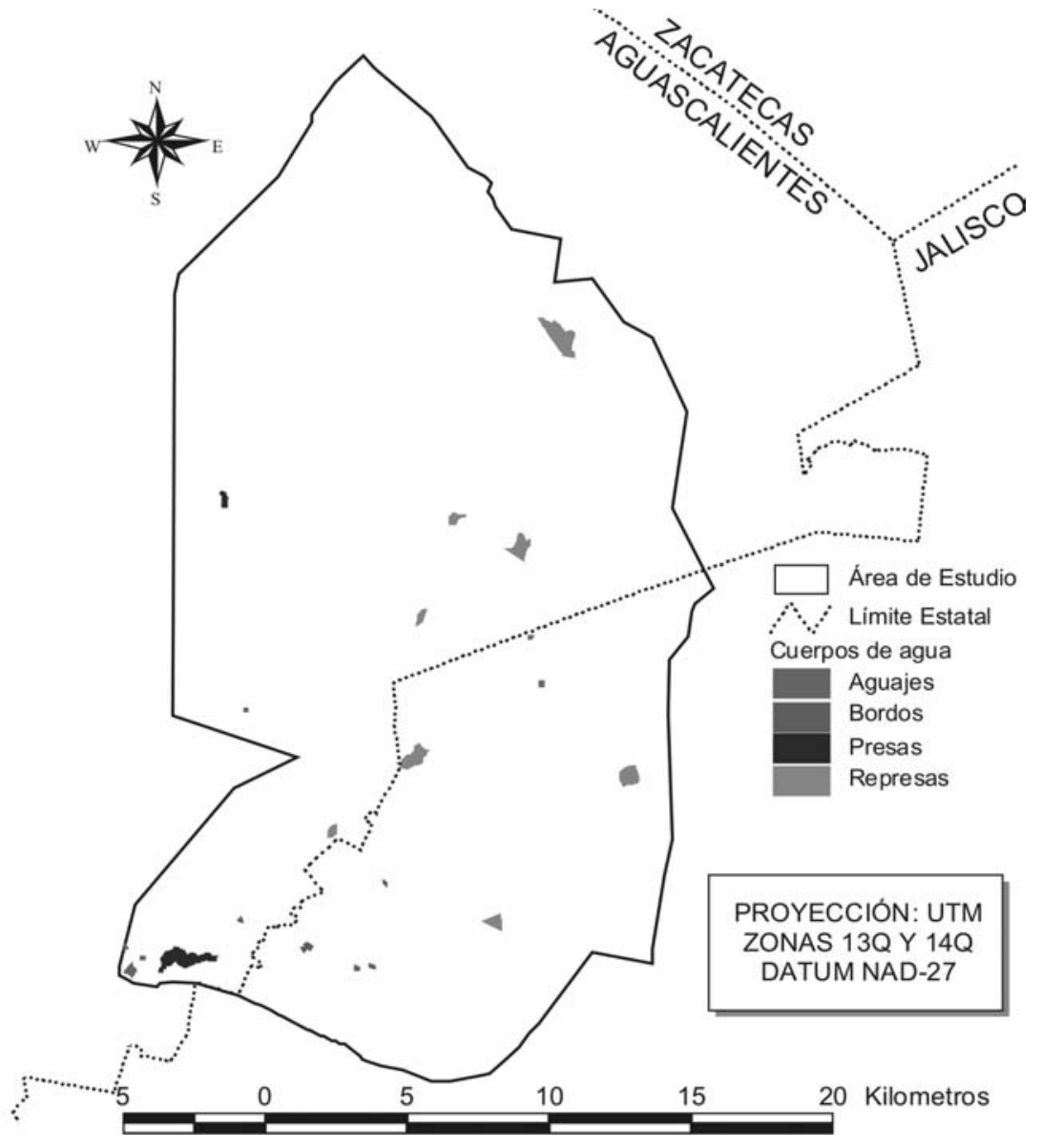

Figura 2

Ubicación de los embalses muestreados. 
geográfica (en áreas de agostadero, agrícolas, de uso múltiple, etc.), se eligieron de manera aleatoria de cinco a ocho embalses por categoría (Fig. 2). Para que el trabajo de campo coincidiera con la época en que la mayor parte de los embalses tienen agua y las aves acuáticas son más abundantes en la zona, se consideraron tres períodos de muestreo; inicio de otoño (de septiembre a octubre de 1994), durante el invierno (de diciembre de 1994 a enero de 1995) y final del invierno (de febrero a marzo de 1995). En promedio, se utilizaron 14 días de muestreo por período para cubrir los embalses seleccionados. Cada uno se visitó por lo menos una vez a la semana en cada período de muestreo. El número muestreado varió de un período a otro debido a que algunos se secaron a lo largo del año (Cuadro 1).

Cuadro 1

Número de embalses estudiados por período de muestreo en la región de El Llano, Aguascalientes-Jalisco, México

\begin{tabular}{lccccc}
\hline \multicolumn{1}{c}{ Período de muestreo } & Bordos & Aguajes & Represas & Presas & Total \\
\hline Septiembre y Octubre de 1994 & 5 & 5 & 8 & 2 & 20 \\
Diciembre de 1994 a Enero de & 7 & 3 & 6 & 2 & 18 \\
1995 & 5 & 2 & 3 & 2 & 12 \\
Febrero y Marzo de 1995 & 17 & 10 & 17 & 6 & 40 \\
Total & & & & \\
\hline
\end{tabular}

Los embalses o cuerpos de agua se clasificaron como sigue:

1) Bordos. Depósitos artificiales de $0.70 \pm 0.44$ ha y con profundidades de entre uno y dos metros, los cuales son utilizados para el almacenamiento de agua extraída por bombeo de pozos construidos para irrigación, generalmente cubiertos de vegetación acuática emergente, como tules (Typha spp) y juncos (Juncus spp).

2) Aguajes. Embalses someros, de menos de un metro de profundidad, construidos para captar agua de lluvia; por lo general, no son mayores de una hectárea de espejo de agua y son utilizados de forma exclusiva como abrevaderos para el ganado. Se ubican principalmente en agostaderos, tienen poca vegetación acuática en sus riberas por efecto del pisoteo y sus aguas generalmente se muestran turbias.

3) Represas. Depósitos artificiales que captan agua de lluvia, generalmente de cuatro hectáreas de espejo de agua o mayores y de menos de dos metros de profundidad. Se utilizan para regar las milpas, como abrevaderos para el ganado después de la cosecha y se ubican, principalmente, en tierras de uso agrícola. Sus riberas presentan varios tipos de vegetación acuática.

4) Presas. Depósitos artificiales de más de seis hectáreas de espejo de agua, provistos de una cortina de piedra, concreto o tierra compactada. Su profundidad es mayor que la de otros cuerpos de agua (más de un metro y medio) y su uso es comparable al tipo anterior. El acceso al ganado doméstico es constante, y no existe vegetación acuática y subacuática en sus riberas. 


\section{Variables consideradas}

En cada uno de los embalses se midió el área de espejo (la superficie cubierta con agua), en hectáreas, empleando un distanciómetro óptico, una brújula Brunton ${ }^{\circledR}$ y una baliza. Con esas herramientas, y caminando por la orilla del agua, se obtuvieron los rumbos y las longitudes que unen los vértices. La superficie cubierta con agua se estimó por triangulación para cada período de muestreo, a través del dibujo de los polígonos en papel milimétrico. En el caso de los bordos de irrigación con pozo, el proceso fue más fácil dado que su forma es rectangular.

Se estimó la densidad de patos (número de individuos por unidad de superficie de espejo de agua), a través del método de Hayne (Davis \& Winstead 1987). En el recuento de las aves participaron tres observadores y se hizo con telescopios Minolta ${ }^{\circledR} 15$ x 60 y binoculares Nikon ${ }^{\circledR} 9$ x 25 , con zoom integrado. En los embalses pequeños (menores de una hectárea de espejo de agua), se contabilizaron todos los individuos presentes, mientras que en los cuerpos de agua de mayor superficie (con más de una hectárea de espejo de agua) se estimó la población desde la orilla a través de transectos de longitud variable $(150 \pm 50 \mathrm{~m})$ o puntos de observación. La elección del método (transectos o puntos) dependió del tamaño de los cuerpos de agua y de la distribución de las aves en el lugar. Mediante la densidad del pato mexicano por tipo de embalse, se obtuvo el número de individuos para cada categoría y período de muestreo.

A una escala mayor se elaboró un inventario de todos los embalses de la región. Para ello se utilizó un Sistema de Información Geográfica construido en una plataforma en ARC VIEW 9.1 ${ }^{\circledR}$, mapas topográficos digitales F13B89, F13D19, F13D29, F14A81 y F14C11 escala 1:50,000, y una imagen satelital del sensor LANDSAT TM 29/45 del mes de agosto de 2002, proporcionados por el departamento de Geomática de la Procuraduría Estatal de Protección al Ambiente de Aguascalientes. Con estos materiales se digitalizaron y clasificaron todos los embalses y se estimó la superficie cubierta con agua de cada embalse. Esta información permitió un análisis de uso-disponibilidad a nivel regional, para determinar sí el patrón de selección de embalses encontrado a nivel de la muestra, se repite al extrapolar las densidades del pato mexicano sobre el universo de los embalses del área de estudio.

\section{Análisis estadístico}

Para determinar sí alguno de los embalses es seleccionado por el pato mexicano, se utilizó un análisis de uso-disponibilidad semejante al utilizado por Neu et al. (1974) y Byers et al. (1984). Este procedimiento requiere de la comparación entre las proporciones del hábitat disponible y del hábitat utilizado. En este estudio la proporción del hábitat disponible correspondió a la superficie cubierta con agua en cada categoría de embalse, mientras que la proporción del hábitat utilizado correspondió a la abundancia absoluta (número de ejemplares) del pato mexicano observados en cada categoría de embalse 
por período de muestreo. De acuerdo con Manly et al. (1993), se empleó un diseño de muestreo tipo I (mediciones realizadas a nivel de poblaciones) y un protocolo de muestreo tipo "A" (muestreo aleatorio del hábitat disponible y hábitat utilizado).

Una vez obtenidos los valores del hábitat utilizado y del hábitat disponible para cada categoría de embalses, se procedió a probar la hipótesis nula de que el pato mexicano utiliza los embalses de acuerdo a su disponibilidad. Dicha hipótesis se analizó con una prueba de Ji-cuadrada de bondad de ajuste, empleando el estadístico $\mathrm{X}_{\mathrm{L}}{ }^{2}$ propuesto por Manly et al. (1993), a un nivel de significancia del 0.01. En los casos en que la hipótesis nula fue rechazada, se estimaron los intervalos de Bonferroni al índice de selectividad $\hat{\mathrm{W}}_{\mathrm{i}}$ de Manly et al. (1993), con un nivel de confianza del 95\%, en donde sí el límite inferior del intervalo es superior a 1 se considera que existe selección, mientras que sí el límite superior del intervalo es inferior a 1 se considera que no la hay.

La magnitud de la selección del pato mexicano por algún tipo de embalse en particular, se estimó con el índice D' de Jacobs (Manly et al. 1993), en el cual el embalse es completamente evitado cuando el índice tiene un valor de -1 mientras que la máxima selección está indicada por +1 .

La extrapolación del número de individuos por tipo de embalse en el primer período de muestreo (bajo el supuesto de que esta temporada coincide con la máxima disponibilidad de agua en la región, y que los embalses muestreados son réplica exacta de los embalses de ese mismo tipo), permitió estimar el número total de individuos. Un nuevo análisis de uso-disponibilidad como el descrito fue aplicado sobre el universo de los embalses para determinar si el pato mexicano sigue presentando el patrón de selección o rechazo encontrado en la muestra de los embalses estudiados.

\section{RESULTADOS}

La mayor cantidad de aves fue observada sobre las represas, durante los tres períodos de muestreo. Una tendencia creciente puede apreciarse conforme progresa la temporada invernal (diciembre 1994 a enero 1995), para disminuir hacia el final del invierno. Por otra parte, la densidad de aves por hectárea de espejo, fue mayor en los bordos de irrigación desde septiembre de 1994 hasta enero de 1995, para declinar considerablemente hacia febrero y marzo de ese mismo año, cuando los embalses con mayor densidad fueron los aguajes (Fig. 4 y 5).

\section{Análisis de uso - disponibilidad sobre la muestra de embalses}

Durante septiembre y octubre de 1994 se observó que los embalses presentaron diferencias en su utilización por el pato mexicano, según su disponibilidad, con lo cual se descartó la hipótesis nula $\mathrm{H}_{0}\left(\mathrm{X}_{\mathrm{L}}{ }^{2}=163.23, \mathrm{gl}=4, \mathrm{p} \leq 0.001\right)$. Los intervalos de confianza 
al índice de selectividad ( $\left.\hat{\mathrm{W}}_{\mathrm{i}}, 99 \%\right)$ mostraron que los bordos $\left(2.33 \leq \hat{\mathrm{W}}_{\mathrm{i}} \leq 9.17\right)$ y las represas $\left(1.28 \leq \hat{\mathrm{W}}_{\mathrm{i}} \leq 1.60\right)$ fueron seleccionados, en tanto que las presas $\left(0.00 \leq \hat{\mathrm{W}}_{\mathrm{i}} \leq\right.$ $0.26)$ fueron evitadas. En contraste, los aguajes fueron utilizados en proporción a su disponibilidad $\left(0.00 \leq \hat{\mathrm{w}}_{\mathrm{i}} \leq 1.56\right)$. En cuanto a la intensidad de selección, el índice de Jacobs (D') mostró que los bordos tuvieron un grado de selección mayor $\left(D^{\prime}=0.72\right)$ que las represas $\left(D^{\prime}=0.37\right)$ y que las presas fueron evitadas $\left(D^{\prime}=-0.82\right)$.

De diciembre de 1994 a enero de 1995 el comportamiento fue semejante. Durante este período, hubo diferencias en la utilización de los embalses según su disponibilidad $\left(\mathrm{X}_{\mathrm{L}}^{2}=460.80, \mathrm{gl}=3, \mathrm{p} \leq 0.001\right)$. Los bordos $\left(3.19 \mathrm{~d} \leq \hat{\mathrm{W}}_{\mathrm{i}} \leq 5.19\right)$ y las represas $\left(1.03 \leq \hat{\mathrm{W}}_{\mathrm{i}}\right.$ $\leq 1.19$ ) fueron seleccionados de acuerdo con los intervalos de confianza del índice de selectividad. Los aguajes continuaron siendo utilizados en proporción a su disponibilidad $\left(0.28 \mathrm{~d} \leq \hat{\mathrm{w}}_{\mathrm{i}} \leq 2.56\right)$. La intensidad de selección fue mayor en los bordos $\left(\mathrm{D}^{\prime}=0.67\right)$ que en las represas $\left(D^{\prime}=0.19\right)$ y las presas, pese a estar disponibles, se dejaron de utilizar evitándose al máximo ( $\left.\mathrm{D}^{\prime}=-1.00\right)$.

Para el período de febrero a marzo de 1995 también se encontró que hubo diferencias en cuanto a la utilización de los embalses según su disponibilidad $\left(\mathrm{X}_{\mathrm{L}}{ }^{2}=93.43, \mathrm{gl}=3, \mathrm{p} \leq\right.$ 0.001 ), pero la tendencia en la selección tuvo un cambio importante. En contraste con los otros períodos de muestreo, los bordos parecieron evitarse de acuerdo con los intervalos de confianza del índice de selectividad $\left(0.00 \leq \hat{\mathrm{W}}_{\mathrm{i}} \leq 0.92\right)$, mientras que la selección de las represas se mantuvo durante este período del año $\left(1.18 \leq \hat{\mathrm{w}}_{\mathrm{i}} \leq 1.40\right)$. Asimismo, los aguajes continuaron mostrando un uso proporcional a su disponibilidad $\left(0.74 \leq \hat{\mathrm{w}}_{\mathrm{i}} \leq 9.42\right)$. La intensidad de selección por las represas fue ligeramente mayor $\left(D^{\prime}=0.59\right)$ que la tendencia a evitar los bordos $\left(D^{\prime}=-0.53\right)$ y las presas continuaron siendo evitadas $\left(\mathrm{D}^{\prime}=-1.00\right)$ por encima de los otros embalses. La información del análisis de uso-disponibilidad en los tres períodos de muestreo se muestra en el Anexo 1.
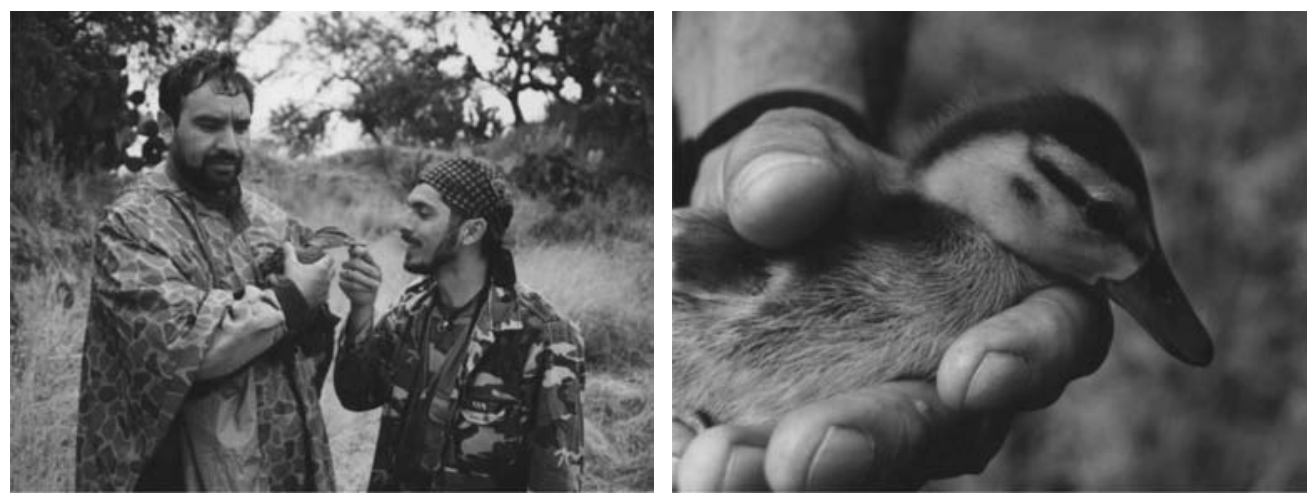

Figura 3

Polluelos de pato mexicano (Anas diazi) observados durante el trabajo de campo. 
Medina-Torres et al.: Pato mexicano en el Llano

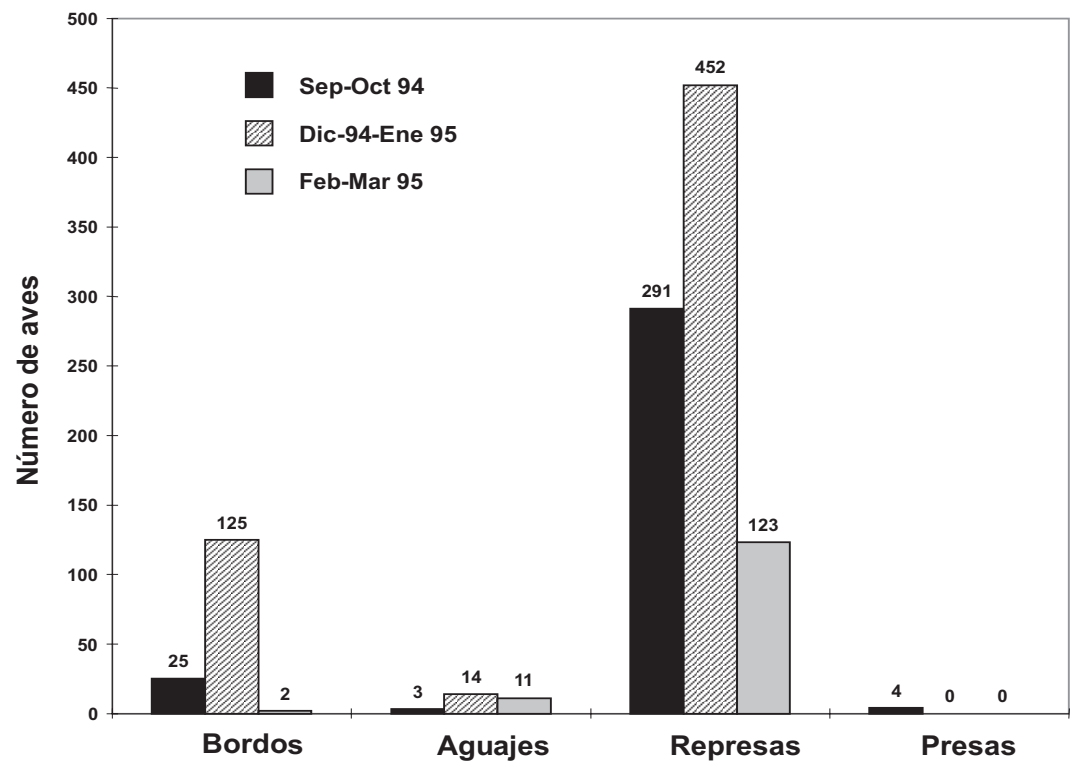

Figura 4

Número de ejemplares de pato mexicano en los embalses estudiados, en la región de El Llano, durante los tres períodos de muestreo.

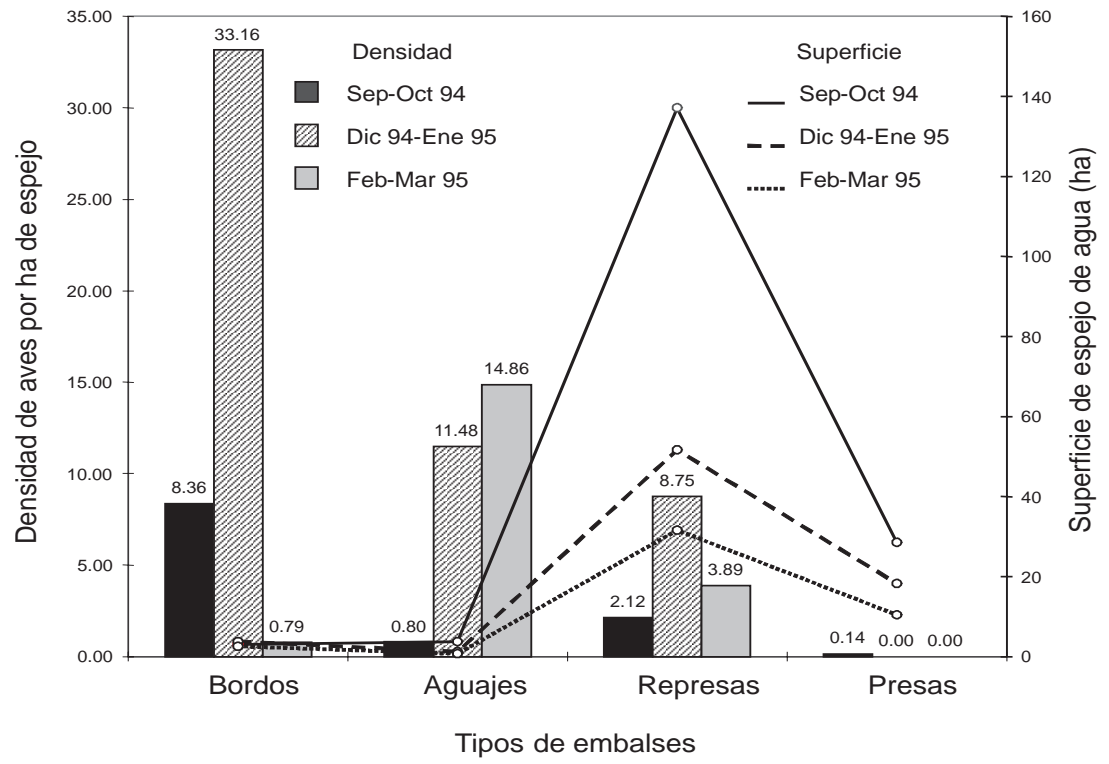

Figura 5

Densidad de pato mexicano durante los tres períodos de muestreo, comparada con la superficie de espejo promedio por tipo de embalse y período de muestreo. 
Si se considera el promedio de los tres períodos de muestreo (Anexo 2), la prueba de hipótesis confirma que al menos uno de los tipos de embalses no fue utilizado de acuerdo a su disponibilidad $\left(\mathrm{X}_{\mathrm{L}}{ }^{2}=294.53, \mathrm{gl}=4, \mathrm{p}_{\leq} 0.001\right)$. Los intervalos de confianza del índice de selectividad confirman la tendencia mostrada durante los dos primeros períodos de muestreo, ya que los bordos $\left(3.57 \leq \hat{\mathrm{W}}_{\mathrm{i}} \leq 8.40\right)$ y las represas $\left(1.30 \leq \hat{\mathrm{W}}_{\mathrm{i}} \leq\right.$ 1.57) fueron los cuerpos de agua seleccionados por el pato mexicano. En cambio, los aguajes fueron utilizados en proporción a su disponibilidad $\left(0.01 \leq \hat{\mathrm{w}}_{\mathrm{i}} \leq 3.59\right)$, y las presas $\left(0.00 \leq \hat{\mathrm{w}}_{\mathrm{i}} \leq 0.01\right)$ fueron evitadas. La intensidad de la selección fue mayor en los bordos $\left(\mathrm{D}^{\prime}=0.74\right)$ que en las represas $\left(\mathrm{D}^{\prime}=0.44\right)$, mientras que las presas fueron altamente evitadas $\left(\mathrm{D}^{\prime}=-0.95\right)$.

\section{Inventario de embalses en el área de estudio}

Del análisis de la información cartográfica y de la imagen satelital se contabilizaron 876 embalses en total, los cuales suman 1,214.59 ha de espejo de agua disponible para el pato mexicano. Las represas fueron los embalses más numerosos $(\mathrm{N}=449)$ y constituyeron cerca del $51.25 \%$ de la superficie de espejo de agua total, mientras que las presas fueron menos abundantes $(\mathrm{N}=2)$ y sólo representaron el $0.25 \%$ del área total cubierta con agua (Cuadro 2).

Cuadro 2

Número y espejo de agua (ha) de los diferentes embalses de la región de El Llano, AguascalientesJalisco, México.

\begin{tabular}{lcc}
\hline Tipo de embalses & \multicolumn{2}{c}{ Área de estudio } \\
& No. de embalses & ha \\
\hline Bordos & $255(29.10)^{1}$ & $177.63(14.62)$ \\
Aguajes & $170(19.40)$ & $148.20(12.21)$ \\
Represas & $449(51.25)$ & $793.36(65.32)$ \\
Presas & $2(00.25)$ & $95.40(07.85)$ \\
Total & 876 & $1,214.59$ \\
\hline
\end{tabular}

En el estado de Aguascalientes se contabilizaron 501 embalses, los cuales cubrieron de manera aproximada una superficie de 681.9 ha, siendo las represas los cuerpos de agua más abundantes con 387 y cobertura de 506.57 ha. En el estado de Jalisco fueron 375 y sumaron un área de 532.69 ha. Los aguajes y los bordos fueron los más numerosos (158 y 155, respectivamente) en esta entidad; sin embargo, las represas $(\mathrm{N}=62)$, a pesar de ser menos abundantes que esos embalses, cubrieron mayor superficie de espejo de agua (286.79 ha), constituyendo el 53.83\% aproximadamente de la superficie de los embalses de la entidad, de manera equivalente, aunque en menor proporción, a lo encontrado en el estado de Aguascalientes (Cuadro 3). 
Medina-Torres et al.: Pato mexicano en el Llano

Cuadro 3

Número y espejo de agua (ha) de los diferentes embalses por entidad federativa en la región de El Llano, Aguascalientes-Jalisco, México.

\begin{tabular}{lcccc}
\hline \multirow{2}{*}{ Tipo de embalses } & \multicolumn{2}{c}{ Aguascalientes } & \multicolumn{2}{c}{ Jalisco } \\
& Cantidad & ha & Cantidad & ha \\
\hline Bordos & $100(19.96)^{1}$ & $63.28(9.28)$ & $155(41.33)$ & $114.35(21.46)$ \\
Aguajes & $12(2.39)$ & $16.65(2.45)$ & $158(42.13)$ & $131.55(24.69)$ \\
Represas & $387(77.25)$ & $506.57(74.28)$ & $62(16.53)$ & $286.79(53.83)$ \\
Presas & $2(0.4)$ & $95.40(13.99)$ & 0 & 0 \\
Total & 501 & 681.9 & 375 & 532.69 \\
\hline
\end{tabular}

Análisis de uso - disponibilidad sobre el universo de embalses

De la abundancia total estimada del pato mexicano y la superficie de espejo de agua de todos los embalses, se encontró una vez más, que al menos un tipo de embalse es utilizado independientemente de su disponibilidad $\left(\mathrm{X}_{\mathrm{L}}{ }^{2}=2659.38, \mathrm{gl}=4, \mathrm{p} \leq \hat{\mathrm{W}}_{\mathrm{i}} \leq 0.001\right)$. En ese análisis, de acuerdo con los intervalos de confianza del índice de selección $\left(3.48 \leq \hat{\mathrm{w}}_{\mathrm{i}}\right.$ $\leq 3.93$ ), los bordos fueron los únicos embalses utilizados en mayor proporción a su disponibilidad ( $\mathrm{D}^{\prime}=0.69$ ). En contraste, los otros tipos de embalses presentaron evidencias de uso por debajo de su disponibilidad ( $\mathrm{IC} \hat{\mathrm{w}}_{\mathrm{i}} \leq 1.000$ ). El índice de Jacobs mostró que las represas fueron evitadas menos que los aguajes $\left(D^{\prime}=-0.48\right)$ y que las presas $\left(D^{\prime}=-0.88\right)$. En tanto que los bordos presentaron una intensidad de selección importante ( $\left.\mathrm{D}^{\prime}=0.69\right)$, sólo superada por la intensidad con que las presas fueron evitadas (Anexo 3).

\section{DISCUSIÓN}

Aún cuando el mayor número de ejemplares de pato mexicano se encontró en las represas, las densidades mayores correspondieron a los bordos de irrigación. Uno de los supuestos básicos que cabe esperar cuando se infiere sobre la posible selección de un hábitat, por una especie, es que ésta será más abundante en el hábitat seleccionado. La mejor prueba de ello, es que la abundancia total estimada a nivel regional entre los bordos de irrigación $(1,506)$, es muy semejante a la que fue estimada para las represas (1,682 aves). Si se considera que el $14.62 \%$ de la superficie de espejo total entre todos los embalses del área de estudio corresponde a bordos de irrigación (1,214.59 ha), resulta evidente que una buena parte de la población del pato mexicano tiende a concentrarse en este tipo de embalses, tal y como ha sido documentado por otros autores (Williams 1980, Lum, 1999, Riojas-López \& Mellink 2005, Moctezuma-Malagón et al. 2007). Una de las características que puede influir en la selección de los bordos, es la persistencia de sus niveles de agua a través del tiempo ya que, en comparación 
con los demás tipos de embalses, éstos han mostrado una mayor estabilidad en cuanto a superficie de espejo y, por tanto, una mayor disponibilidad de agua.

Los resultados del análisis de uso-disponibilidad destacan la importancia de los embalses utilizados para almacenar el agua extraída por bombeo con fines agrícolas como hábitat para el pato mexicano. Esto apoya lo afirmado por otros autores (Williams 1980, Scott \& Reynolds 1984, Cisneros 1985, Leopold 1990, Pérez-Arteaga et al. 2002, Riojas-López \& Mellink 2005), que citan además que esos embalses son de gran importancia para la conservación de dicha especie. Es conveniente señalar que, a pesar de que los embalses donde se recolectó la información representan una pequeña fracción de los obtenidos de la imagen satelital, la mayor parte de ellos fueron utilizados de manera superior a lo esperado, en particular durante las dos primeras temporadas de muestreo, correspondientes al otoño y el inicio de invierno. Una posible interpretación ecológica de esta selección, se relaciona con el hecho de que casi en todos los bordos se encontraron polluelos de pato mexicano (Fig. 3) y ello pudiera indicar que este tipo de embalses representa un hábitat adecuado para la reproducción y crianza de la especie. Williams (1980) menciona que la presencia de plantas acuáticas como tules (Typha spp) y juncos (Juncus spp), proporcionan a los polluelos del pato mexicano sitios con una buena cobertura vegetal que los protege de los depredadores. Los cuerpos de agua en los que existe vegetación emergente, representada principalmente por tule, favorece la presencia de una gran variedad de especies de aves entre las que sobresale, por su número, el pato mexicano (Lum 1999).

La tendencia en el uso de esos cuerpos de agua se mantuvo durante el invierno (diciembre y enero), lo que pudo deberse a que los individuos juveniles del pato mexicano continuaron su desarrollo bajo la protección de los tulares, según pudo constatarse durante el estudio. Sólo al final de la temporada invernal (febrero y marzo) los bordos fueron evitados, posiblemente como consecuencia del incremento en la actividad cinegética que se lleva a cabo durante esos meses del año, de manera particular en esos embalses, según se desprende de otros trabajos realizados en el área de estudio. Como ejemplo, Medina (1996) encontró que los cazadores prefieren cazar en los bordos. Paradójicamente, la gran cobertura de los tulares en los bordos, que ofrece las condiciones adecuadas para el desarrollo de los polluelos del pato mexicano, es utilizada por los cazadores para aproximarse sin ser vistos y con ello incrementar sus expectativas de una caza exitosa. Durante el trabajo de campo se encontraron grupos de cazadores y evidencias de caza en la mayoría de los bordos visitados, particularmente al final del invierno.

Por otra parte, la preferencia del pato mexicano por embalses de aguas someras es un hecho demostrado y ampliamente documentado por varios autores (Bellrose 1976, Williams 1980, Cisneros 1985, Leopold 1990). Lo anterior coincide con el hecho de que las represas (con aguas poco profundas) registraron un uso superior al esperado durante 
los tres períodos de muestreo. Así mismo, las represas fueron los únicos cuerpos de agua seleccionados al término de la temporada invernal (febrero y marzo). Lo anterior pudo deberse a que los individuos juveniles de pato mexicano, ya con plena capacidad de vuelo y junto con las aves adultas, comenzaron a dispersarse buscando los sitios con aguas someras y con cobertura vegetal adecuada, para comenzar un nuevo ciclo de cortejo y apareamiento.

Una interpretación ecológica de la aparente evasión de los aguajes y las presas puede fundarse en las características de esos sitios. En el caso de los aguajes, se encontró que son sitios de poca extensión y con poca vegetación, ya que el libre acceso al ganado doméstico a estos sitios no ha permitido su desarrollo. Otra característica que puede influir en este rechazo es la calidad del agua. En estos sitios, el agua invariablemente se encontró revuelta con sedimentos suspendidos, excretas y orina de ganado, lo que posiblemente no hace de estos sitios un buen lugar para el pato mexicano. Debe considerarse además que estos cuerpos de agua son los menos abundantes, por lo que su disponibilidad es baja. En el caso de las presas se encontraron características semejantes a los aguajes, escasa vegetación ribereña y estar expuestos a un constante pisoteo por el ganado doméstico.

Al considerar el inventario total de los cuerpos de agua y la población estimada del pato mexicano se observa un resultado ligeramente distinto, pero que de alguna manera confirma la importancia de los embalses generados por el desarrollo agrícola de la zona. En este caso, los bordos fueron los únicos cuerpos de agua que presentaron evidencias de selección por el pato mexicano. El aparente rechazo de las represas debe tomarse con cautela, ya que se tiene la incertidumbre de que el agua existente durante el muestreo en esos sitios correspondiera fielmente con el área de espejo determinada por medio del sistema de información geográfica, cosa que no sucede con los bordos de irrigación, que por lo general no varían en cuanto a su disponibilidad de agua.

El hecho de que los bordos sean seleccionados por el pato mexicano permite anticipar que esos embalses podrían constituir el hábitat critico para la reproducción y conservación de dicha especie; sobre todo, ante la disminución y contaminación de los principales humedales del Altiplano Central Mexicano, como el Lago de Chapala y la Cuenca del Río Lerma, donde se encuentra una de las mayores poblaciones de esta ave (Perez-Arteaga et al. 2002).

Una medida adecuada para la protección y conservación del pato mexicano sería excluir a los bordos de la actividad cinegética, ya que son ampliamente utilizados para dicha actividad. Un uso alternativo de los bordos se ha documentado por Moctezuma-Malagón et al. (2007), quienes abordan el uso integral de estos embalses para el policultivo de especies autóctonas en el centro de México, en el 
cual se demuestra la importancia de los bordos de irrigación, con un ejemplo de crianza de pato mexicano en estanques de riego. En contraste, sería adecuado establecer áreas de caza de aves acuáticas en las represas, en donde la cantidad de aves podría ser suficiente para satisfacer la demanda de los cazadores. Colateralmente a estas medidas, sería conveniente desarrollar un programa de mejoramiento de hábitat en los aguajes mediante la construcción de cercos que impidan el libre acceso del ganado, instalando además bebederos para que los animales domésticos puedan abrevar libremente sin dañar la cubierta vegetal de las riberas y de los embalses. Estas acciones permitirían modificar las características del hábitat, de tal manera que también pudieran servir como sitios de protección para el pato mexicano.

Por otra parte, se ha encontrado que una gran cantidad de represas pequeñas están construidas en terrenos de cultivo y que son utilizadas para proporcionar riego de auxilio a los cultivos de temporal durante la época de siembra y como abrevadero para el ganado después de las cosechas. Esos sitios también pueden ser objeto de mejora, como en el caso de los aguajes, y con ello incrementar la cantidad y calidad de hábitat disponible para que el pato mexicano pueda reproducirse de manera exitosa en la región de El Llano, sin que la caza atente contra la renovación de la población. Por último, se sugiere orientar futuros estudios de selección de recursos a un nivel más detallado, para determinar qué factores o atributos del hábitat influyen para que los cuerpos de agua sean utilizados por estos anátidos. Un programa de fomento a nivel regional para el mantenimiento de los pequeños embalses generados por el desarrollo agrícola y pecuario de la región, en combinación con actividades de turismo cinegético, podrían contribuir a diversificar la economía rural de los habitantes de El Llano, además de permitir otros usos alternativos y sostenibles para el agua.

\section{AGRADECIMIENTOS}

El primer autor agradece al Colegio de Postgraduados, al CONACYT, al Instituto del Medio Ambiente del Estado de Aguascalientes (antes Subsecretaría de Ecología), y a la Procuraduría Estatal de Protección al Ambiente por las facilidades y apoyos otorgados para la realización de esta investigación.

\section{LITERATURACITADA}

Aldrich, J.W. \& K.P Baer. 1970. Status and speciation in the Mexican duck (Anas diazi). Wilson Bull. 82:63-73.

Arriaga-Cabrera, L., V. Aguilar-Sierra \& J. Alcocer-Duran. 2000. Aguas continentales y diversidad biológica de México. CONABIO. México, D.F. 327 p. 
Bellrose, F.C. 1976. Ducks, Geese and Swans of North America. Wildlife Management Institute. Washington, D.C. 540 p.

Byers, C. R., R. K. Steinhorst \& P. R. Krausman. 1984. Clarification of a technique for analysis of utilization-availability data. J. Wildl. Manage. 48:1050-1053.

Cisneros, T. J. E. 1985. Mini-hábitat: Estrategia para la Conservación del Pato Mexicano. Primer Simposio Internacional de Fauna Silvestre. México, DF. pp 957-965.

Davis, D.E. \& R.L. Winstead. 1987. Estimación de tamaños de población de vida silvestre. Pp. 233-258. In: T.R. Rodríguez (Ed.). Manual de técnicas de gestión de vida silvestre. The Wildlife Society. Washington, D.C.

DOF. 2002. Norma Oficial Mexicana (NOM-059-ECOL-2001). Protección ambiental-Especies nativas de México de flora y fauna silvestres-Categorías de riesgo y especificaciones para su inclusión, exclusión o cambio-Lista de especies en riesgo. Secretaría de Medio Ambiente y Recursos Naturales. Segunda sección. 6 de marzo de 2002. México, D.F. p. 22.

Grenot, C.J. 1983. Desierto Chihuahuense. Fauna del Bolsón de Mapimí. Universidad Autónoma Chapingo. Depto. de Zonas Áridas. Chapingo, México. 63 p.

Howell, S.N.G. \& S. Webb. 1995. A guide to the birds of Mexico and Northern Central America. Oxford University Press. New York. $851 \mathrm{p}$.

Hubbard, J.P. 1977. The biological and taxonomic status of Mexican duck. New Mexico Dept. Game and Fish Bull. 16:1-56.

INE. 1994. Plan de Manejo de Aves Acuáticas de Norteamérica: Extensión del compromiso. Instituto Nacional de Ecología. México, D.F. 45 p.

INEGI. 1981. Síntesis Geográfica de Aguascalientes. México, D.F. 98 p. . 1993. Estudio Hidrológico del Estado de Aguascalientes. Aguascalientes. 164 p.

Kaufman, K. 2005. Guía de campo a las aves de Norteamérica. Traducida por Patricia Manzano Fischer. Houghton Mifflin Company. New York, N.Y. 392 p.

Leopold, A.S. 1990. Fauna silvestre de México. Editorial Pax. México, D.F. 608 p.

Lum, K. 1999. Los humedales: elemento clave de la respuesta a la crisis del agua. En: La Jornada Ecológica. Suplemento de Abril, 1999. http://www.laneta.apc.org/emis/jornada/abril99/ index.htm.

McCraken, K.G., W.P., Johnson \& F.H., Sheldon. 2001. Molecular population genetics, phytogeography, and conservation biology of the mottled duck (Anas fulvigula). Conservation Genetics 2:87-102.

Manly, B., L. McDonald \& D. Thomas. 1993. Resource Selection by Animals. Statistical design and analysis for field studies. Chapman \& Hall. London. $177 \mathrm{p}$.

Medina, S. 1996. Distribución, abundancia y uso de hábitat de patos y gansos en la región de El Llano, Aguascalientes. Tesis de Maestría. Especialidad de Ganadería. Colegio de Postgraduados. $110 \mathrm{p}$.

Mellink, E. 1994. Grazing and Mexican ducks in central Mexico. Euphonia 3:5-7.

Moctezuma-Malagón, A., G. de la Lanza-Espino, C. González-Rebeles Islas and C. GonzálezEsquivel. 2007: Multiculture of native species in irrigation ponds in central Mexico. Livestock Research for Rural Development. Volume 19, Article \#14. En: http://www.cipav.org.co/lrrd/ lrrd19/1/moct19014.htm. 
Neu, W. C., R. C. Byers \& M. J. Peek. 1974. A technique for analysis of utilization-availability data. J. Wildl. Manage. 38:541-545.

Ohlendorf, H.M \& R.F. Patton. 1971. Nesting record of Mexican duck (Anas diazi) in Texas. Wilson Bull. 83:97.

Palmer, R.S. 1976. Handbook of North American Birds. Waterfowl (first part). Yale University Press. New Haven, Connecticut 2:309-313.

Peek, J. M. 1986. A review of Wildlife Management. Prentice-Hall, Englewood Cliffs, New Jersey. $486 \mathrm{p}$.

Perez-Arteaga, A. \& K.J., Gaston. 2004. Status of American coot Fulica americana (Gruiformes: Rallidae) wintering in Mexico. Acta Zool. Mex. (n.s.) 20:253-263.

Perez-Arteaga, A., K.J., Gaston \& M., Kershaw. 2002. Population trends and priority conservation sites for Mexican duck Anas diazi. Bird Conserv. Int. 12:35-52.

Peterson, R.T. \& E.L., Chalif. 1989. Aves de México. Traducido por Mario Ramos y Maria Isabel Castillo. Diana, México, D.F. 473 p.

Riojas-López, M. E. \& E. Mellink. 2005. Potential for biological conservation in man-modified semiarid habitats in northeastern Jalisco, Mexico. Biodiversity and Conservation 14: 22512263.

Scott, N.J., Jr. \& R.P. Reynolds. 1984. Phenotypic variation of the Mexican duck (Anas phatyrhynchos diazi) in Mexico. Condor 86:266-274.

SEDUE. 1989. Guía de aves acuáticas cinegéticas de México. Secretaría de Desarrollo Urbano y Ecología. Subsecretaría de Ecología, México, D.F. 54 p.

Tomlinson, R.E., S.H. Levy \& J.J. Levy. 1973. New distributional records of breeding Mexican ducks. Condor 75:120-121.

Williams, S.O. 1980. The Mexican Duck in Mexico: Natural History, Distribution, and Population Status. Ph.D. Thesis. Colorado State University. Fort Collins, Colorado. 284 p.

Recibido: 22 de febrero 2006

Aceptado: 9 de marzo 2007 
Anexo 1

Uso de embalses por el pato mexicano según su disponibilidad en la región de El Llano, Aguascalientes-Jalisco, México, por período de muestreo.

\begin{tabular}{|c|c|c|c|c|c|c|c|}
\hline Atributos & $\begin{array}{c}\text { Hábitat } \\
\text { disponible } \\
\text { (ha) }\end{array}$ & $\begin{array}{r}\text { Proporción } \\
\text { de hábitat } \\
\text { disponible }\end{array}$ & $\begin{array}{c}\text { Hábitat } \\
\text { utilizado } \\
\text { observado } \\
\text { (numero de } \\
\text { patos) }\end{array}$ & $\begin{array}{l}\text { Proporción } \\
\text { de hábitat } \\
\text { utilizado }\end{array}$ & \multicolumn{2}{|c|}{$\begin{array}{l}\text { Intervalo de Confianza } \\
\text { Bonferroni del Índice de } \\
\text { Selectividad } \\
\left(\hat{\mathrm{w}}_{\mathrm{i}}=\hat{\mathrm{o}}_{\mathrm{i}} / \pi_{\mathrm{i}}\right)\end{array}$} & $\begin{array}{c}\text { Índice de } \\
\text { Jacobs }\end{array}$ \\
\hline \multicolumn{8}{|c|}{ Septiembre y Octubre de 1994} \\
\hline Embalses & $m_{i}$ & $\pi_{i}$ & $u_{i}$ & $\hat{o}_{i}$ & Inferior & Superior & $D_{i}$ \\
\hline Bordos & 2.99 & 0.01 & 25.00 & 0.05 & 2.33 & 9.17 & +0.72 \\
\hline Aguajes & 3.75 & 0.01 & 3.00 & 0.01 & 0.00 & 1.56 & $=$ \\
\hline Represas & 137.10 & 0.44 & 291.00 & 0.62 & 1.28 & 1.60 & +0.37 \\
\hline Presas & 28.58 & 0.09 & 4.00 & 0.01 & 0.00 & 0.26 & -0.82 \\
\hline
\end{tabular}

$\mathrm{X}_{\mathrm{L}}^{2}=163.23, \mathrm{gl}=4, \mathrm{p} \leq 0.001$

Diciembre de 1994 a Enero de 1995

$\begin{array}{lrrrcccc}\text { Embalses } & m_{i} & \pi_{i} & u_{i} & \hat{o}_{i} & \text { Inferior } & \text { Superior } & D_{i} \\ \text { Bordos } & 3.77 & 0.05 & 125.00 & 0.21 & 3.19 & 5.19 & +0.67 \\ \text { Aguajes } & 1.22 & 0.02 & 14.00 & 0.02 & 0.28 & 2.56 & = \\ \text { Represas } & 51.68 & 0.69 & 452.00 & 0.77 & 1.03 & 1.19 & +0.19 \\ \text { Presas } & 18.24 & 0.24 & 0.00 & 0.00 & 0.00 & 0.00 & -1.00\end{array}$

$\mathrm{X}_{\mathrm{L}}{ }^{2}=460.80, \mathrm{gl}=3, \mathrm{p} \leq 0.001$

Febrero y Marzo de 1995

\begin{tabular}{lccccccc} 
Embalses & $m_{i}$ & $\pi_{i}$ & $u_{i}$ & $\hat{o}_{i}$ & Inferior & Superior & $D_{i}$ \\
Bordos & 2.54 & 0.06 & 2.00 & 0.02 & 0.00 & 0.92 & -0.53 \\
Aguajes & 0.74 & 0.02 & 11.00 & 0.08 & 0.74 & 9.42 & $=$ \\
Represas & 31.59 & 0.70 & 123.00 & 0.90 & 1.18 & 1.40 & +0.59 \\
Presas & 10.42 & 0.22 & 0.00 & 0.00 & 0.00 & 0.00 & -1.00 \\
$\mathrm{X}_{\mathrm{L}}{ }^{2}=93.43, \mathrm{gl}=3, \mathrm{p} \leq 0.001$ & & & & & & \\
\hline
\end{tabular}

+ indica selección; - indica evasión; = indica uso proporcional a disponibilidad 
Anexo 2

Uso de embalses por el pato mexicano según su disponibilidad en la región de El Llano, Aguascalientes-Jalisco, México, al considerar el promedio de los tres períodos de muestreo.

Promedio Septiembre 1994 -Marzo 1995

\begin{tabular}{lccccccc}
\hline Atributos & $\begin{array}{c}\text { Hábitat } \\
\text { disponible } \\
\text { (ha) }\end{array}$ & $\begin{array}{c}\text { Proporción } \\
\text { de hábitat } \\
\text { disponible }\end{array}$ & $\begin{array}{c}\text { Hábitat } \\
\text { utilizado } \\
\text { observado } \\
\text { (numero de } \\
\text { patos) }\end{array}$ & $\begin{array}{c}\text { Proporción } \\
\text { de hábitat } \\
\text { utilizado }\end{array}$ & $\begin{array}{c}\text { Intervalo de Confianza } \\
\text { Bonferroni del Índice de } \\
\text { Selectividad } \\
\left(\hat{\mathbf{w}}_{\mathbf{i}}=\hat{\mathbf{o}}_{\mathbf{i}} / \pi_{\mathbf{i}}\right)\end{array}$ & $\begin{array}{c}\text { Índice de } \\
\text { Jacobs }\end{array}$ \\
\hline Embalses & $m_{i}$ & $\pi_{i}$ & $u_{i}$ & $\hat{o}_{i}$ & Inferior & Superior & $D_{i}$ \\
Bordos & 3.10 & 0.02 & 51.00 & 0.13 & 3.57 & 8.40 & +0.74 \\
Aguajes & 1.90 & 0.01 & 9.00 & 0.02 & 0.01 & 3.59 & $=$ \\
Represas & 73.46 & 0.51 & 288.00 & 0.73 & 1.30 & 1.57 & +0.44 \\
Presas & 19.08 & 0.13 & 1.00 & 0.00 & 0.00 & 0.01 & -0.95 \\
$\mathrm{X}_{\mathrm{L}}{ }^{2}=294.53, \mathrm{gl}=4, \mathrm{p} \leq 0.001$ & & & & & & \\
\hline
\end{tabular}

+ indica selección; - indica evasión; = indica uso proporcional a disponibilidad

Anexo 3

Uso de embalses por el pato mexicano según su disponibilidad en la región de El Llano, Aguascalientes-Jalisco, México, al considerar la población estimada total para SeptiembreOctubre de 1994, sobre el inventario de cuerpos de agua.

\begin{tabular}{lccccccc}
\hline Atributos & $\begin{array}{c}\text { Hábitat } \\
\text { disponible } \\
\text { (ha) }\end{array}$ & $\begin{array}{c}\text { Proporción } \\
\text { de hábitat } \\
\text { disponible }\end{array}$ & $\begin{array}{c}\text { Hábitat } \\
\text { utilizado } \\
\text { observado } \\
\text { (numero de } \\
\text { patos) }\end{array}$ & $\begin{array}{c}\text { Proporción } \\
\text { de hábitat } \\
\text { utilizado }\end{array}$ & $\begin{array}{c}\text { Intervalo de Confianza } \\
\text { Bonferroni del Índice } \\
\text { de Selectividad } \\
\left(\hat{\mathbf{w}}_{\mathbf{i}}=\hat{\mathbf{o}}_{\mathbf{i}} / \pi_{\mathrm{i}}\right)\end{array}$ & $\begin{array}{c}\text { Índice de } \\
\text { Jacobs }\end{array}$ \\
\hline Embalses & $m_{i}$ & $\pi_{i}$ & $u_{i}$ & $\hat{o}_{i}$ & Inferior & Superior & $D_{i}$ \\
Bordos & 178 & 0.11 & 1,506 & 0.40 & 3.48 & 3.93 & +0.69 \\
Aguajes & 148 & 0.09 & 126 & 0.03 & 0.27 & 0.47 & -0.48 \\
Represas & 793 & 0.48 & 1,682 & 0.45 & 0.88 & 0.98 & -0.07 \\
Presas & 95 & 0.06 & 15 & 0.01 & 0.01 & 0.12 & -0.88 \\
$\mathrm{X}_{\mathrm{L}}{ }^{2}=2659.38, \mathrm{gl}=4, \mathrm{p} \leq 0.001$ & & & & & & & \\
\hline
\end{tabular}

+ indica selección; - indica evasión; = indica uso proporcional a disponibilidad 
